\title{
Kidney and Pancreas Transplantation in the United States, 1995-2004
}

\author{
D. J. Cohen ${ }^{a, *}$, L. St. Martin ${ }^{b}$, L. L. Christensen ${ }^{c}$, \\ R. D. Bloom ${ }^{d}$ and R. S. Sunge \\ ${ }^{a}$ Columbia University Medical Center, New York, NY, USA \\ ${ }^{b}$ Department of Health and Human Services, Health \\ Resources and Services Administration, Healthcare \\ Systems Bureau, Division of Transplantation, Rockville, \\ $M D, U S A$ \\ 'Scientific Registry of Transplant Recipients, University \\ Renal Research and Education Association, Ann Arbor, \\ MI, USA \\ ¿University of Pennsylvania, Philadelphia, PA, USA \\ e Scientific Registry of Transplant Recipients, University of \\ Michigan, Ann Arbor, MI, USA \\ *Corresponding author: David J. Cohen, \\ djc5@columbia.edu
}

This article examines OPTN/SRTR data on kidney and pancreas transplantation for 2004 and the previous decade, and discusses recent changes in kidneypancreas (KP) allocation policy and emerging issues in kidney donation after cardiac death (DCD). Although the number of kidney donors continues to increase, new waiting list registrations again outpaced the number of kidney transplants performed, rising by $11 \%$ between 2003 and 2004 and contributing to a 1-year increase of $8 \%$ in the number of patients active on the waiting list. DCD has increased steadily since 2000; 39\% more DCD transplants were performed in 2004 than 2003. Both deceased donor and living donor kidney graft survival rates remain excellent and are improving. The number of people living with a functioning kidney transplant doubled between 1995 and 2004, to 101440 with a functioning kidney-alone and 7213 with a functioning KP. Health care providers in all settings are more likely to be exposed to these transplant recipients. Patient survival following simultaneous pancreas-kidney (SPK) transplantation is excellent and has improved incrementally since 1995; death rates in the first year fell from 60 per 1000 patient-years at risk in 2001 to 45 in 2003 . The number of solitary pancreas transplants increased dramatically in 2004.

Note on sources: The articles in this report are based on the reference tables in the 2005 OPTN/SRTR Annual Report, which are not included in this publication. Many relevant data appear in the figures and tables included here; other tables from the Annual Report that serve as the basis for this article include the following Tables 1.1, 1.3 1.7, 1.8, 5.1-5.14, 6.1-6.6, 6.9, 6.12, 7.1-7.7, 7.9, $7.12,8.1-8.4,8.6,8.7$ and 8.9-8.14. All of these tables may be found online at http://www.ustransplant.org
Key words: Deceased donors, graft survival, kidney transplantation, kidney-pancreas transplantation, living donors, organ donation, pancreas transplantation, patient survival, SRTR, waiting list

\section{Introduction}

This article reviews kidney and pancreas transplantation data for 2004 and the past decade, covering trends in kidney-alone, simultaneous pancreas-kidney (SPK), pancreas after kidney and isolated pancreas transplantation. We also look at recent changes in kidney-pancreas (KP) allocation policy and emerging issues in kidney donation after cardiac death (DCD). Unless otherwise noted, the statistics in this article are drawn from the reference tables in the 2005 OPTN/SRTR Annual Report. A companion article in this report, 'Analytical Methods and Database Design: Implications for Transplant Researchers, 2005,' explains the methods of data collection, organization and analysis that serve as the basis for this article (1). Additional details on the methods of analysis employed herein may be found in the reference tables themselves or in the Technical Notes of the OPTN/SRTR Annual Report, both available online at http://www.ustransplant.org.

\section{Kidney Transplantation}

\section{The kidney transplant waiting list}

The number of patients waiting for a deceased donor kidney transplant continues its relentless increase. At the end of 2004, there were 57910 patients on the kidney-alone transplant waiting list. This represents an increase of $8 \%$ since the end of 2003 and is approximately double the number of waitlisted patients in 1995. The number of patients active on the waiting list at the end of 2004 was 45676 , whereas there were only 25509 patients active on the list at the end of 1995, a $79 \%$ increase. (A patient active on the waiting list does not have any contraindications to transplantation at the current time and is actively awaiting transplantation. Patients can be placed on inactive status if they temporarily are not appropriate candidates for transplantation.) There is no indication that these trends will change in the near future. However, while the prevalence of end-stage renal disease (ESRD) has been increasing in the general population, the rate of increase actually slowed 
during the period 1992-2002, with a rate of increase of only $2.5 \%$ in 2002 (2).

The number of new registrations continues to grow more rapidly than the number of transplants performed. There were 27131 new kidney-alone waiting list registrations in 2004, an increase of $11 \%$ since 2003 and $52 \%$ since 1995. By comparison, the number of kidney transplants performed increased only $43 \%$ over this same time period. The greatest numbers of new registrants were in the 50-64 year-old age range, followed by, in decreasing order, the group aged 35-49 years, the group aged 18-34 years and the group aged 65 years and older. However, the age groups with the greatest percentage increase in registrations since 2003 were the group aged 65 years and older (20\%) and the group aged $50-64$ years (15\%).

The age distribution of active waiting list registrants has continued its shift toward greater representation of older registrants. The percentage of children under age 18 remained stable over the past 10 years at $2 \%$ of the list, while there was a continued slow decline in the percentages of active waiting list patients aged $18-34$ and $35-49$ years, from $21 \%$ and $40 \%$ in 1995 to $13 \%$ and $31 \%$ in 2004 . In parallel, patients aged 50 years and older accounted for $55 \%$ of patients on the active waiting list at the end of 2004 , compared to $37 \%$ at the end of 1995 . At the end of 2004 , the percentage of patients aged 65 years and older, at $14 \%$, slightly exceeded the percentage aged $18-34$ years for the first time since 1995. Several factors likely contributed to this growth in representation of the older age groups, including the aging of the general population and the increased incidence of ESRD with aging, and advances in transplantation that have made it possible for transplant centers to now consider transplantation as a viable option for patients with advanced age and comorbidities.

African Americans made up 35\% of the active waiting list at the end of 2004, while whites made up 39\%. This continues to be far in excess of the proportion of African Americans in the total U.S. population (12\%) (3). While the percentage of African Americans active on the waiting list has remained stable over the past decade, the percentage of whites has decreased by about $10 \%$ as representation of Hispanics and Asians has increased. Representation by sex has been relatively unchanged over the past decade, with males representing about $58 \%$ and females about $42 \%$ of the active waiting list. The representation of $A B O$ blood groups also has been stable over the past decade; more than half of the patients on the active waiting list have blood type O. Over the past decade, while the absolute number has doubled and continues to increase steadily, the percentage of waiting list patients who had had a previous transplant of any organ declined, from $23 \%$ in 1995 to $19 \%$ in 2004 . Similarly, the percentage of patients who had had a previous kidney or KP transplant declined by about $5 \%$. Only about $1 \%$ of active waiting list patients are nonresident aliens.
Approximately $21 \%$ of patients on the waiting list at the end of 2004 were inactive, compared to $14 \%$ for the period 2000-2004. This increase likely reflects the change in the Organ Procurement and Transplantation Network (OPTN) policy (approved June 2003), which now allows patients to continue to accrue waiting time points while on inactive status. As a result of the policy, transplant centers are more likely to place patients who are temporarily medically unsuitable for transplantation on inactive status, since they will not lose waiting time. Of note, patients with longer waiting times appear far more likely to be inactive: at the end of $2004,48 \%$ of inactive patients had waiting times of more than 3 years, and $24 \%$ waited more than 5 years, compared with $22 \%$ and $7 \%$ of active patients. Diabetics are no more likely than non-diabetics to have inactive status. The ultimate suitability for transplantation of these inactive patients with long waiting times is unknown.

The percentage of patients who remain on the waiting list multiple years continued to increase. Twenty-two percent of active waiting list patients at the end of 2004 had been waiting 3 years or more, compared to just $14 \%$ at the end of 1995 . The percentage of patients waiting less than 1 year declined over the previous decade, from $48 \%$ to $38 \%$ of the active waiting list. Approximately $60 \%$ of waiting list patients had a panel reactive antibody (PRA) level less than $10 \%$, compared to only $49 \%$ in 1995 , while those with a PRA greater than $80 \%$ decreased from $20 \%$ to $13 \%$. Glomerular disease, diabetes and hypertension remain the most common primary diagnoses among active waiting list patients, at $22 \%, 27 \%$ and $20 \%$, respectively. According to data from the U.S. Renal Data System, the rate of incident diabetic ESRD appears to be leveling off (2). However, it is likely that diabetes will remain among the most common primary diagnoses of waiting list patients in years to come. The percentage of patients with a primary diagnosis of nephrosclerosis doubled between 1995 and 2004, and the percentage of patients with polycystic kidney disease (PKD) remained steady at just over 6\%. This represents a somewhat different distribution of causes of ESRD compared with the total prevalent ESRD population in the United States, with a reduced proportion of patients with diabetes and hypertension and an increase in those with glomerular disease and PKD. This is likely related to comorbidities present in many diabetics and hypertensives, which preclude renal transplantation as an option.

Waiting times have increased to such a degree that overall median time to transplant for new waiting list registrants cannot be calculated for entrants after 2001; that year, the median time to transplant was 1176 days. Over the past decade, there has been considerable variability in median time to transplant for new kidney waiting list registrants under age 5; the small numbers of registrants coupled with the relative lack of available donors of appropriate size, quality, blood type or human leukocyte antigen $(H L A)$ match would account for this. Among registrants aged 6 years and older, there is a clear trend of increasing 
waiting times since 1995, which is most pronounced among adults aged 50-64 years. In November 2004, the kidney allocation policy was changed to eliminate pediatric time goals and give patients under age 18 high priority for allocation of kidneys from donors less than 35 years old; whether this change will, as intended, reduce waiting times for children remains to be seen. The median time to transplant for adolescents aged 11-17 years who were waitlisted in 1999 (the most recent year for which this figure could be calculated) was 349 days, compared to 1600 days for registrants aged 65 years and older. The median time to transplant for ethnic minorities was considerably greater than that for whites. For African American registrants added to the list in 1999, the median time to transplant was 1822 days; for Hispanics/Latinos, 1321 days; for Asians, 1537 days; and for whites, 758 days.

The median time to transplant remains longest for blood group B registrants, at 1754 days for registrants listed in 1999 , and is shortest for blood group $A B$ registrants listed the same year, at 396 days. Increased use of blood group $A 2$ and $A 2 B$ kidneys in blood group $B$ registrants could potentially reduce waiting times for blood group $B$ registrants (4). The median time to transplant for registrants with a previous organ transplant is nearly twice as long as that for registrants waiting for a first transplant, likely reflecting sensitization and the presence of comorbidities. Nonresident aliens have a median time to transplant similar to U.S. residents. As might be expected, the median time to transplant increases with increasing PRA: 919 days (PRA 0-9\%) versus 1638 days (PRA 10-79\%) for those listed in 2000. There were too few recipients who had PRAs greater than $80 \%$ or who were listed after 2000 to calculate median waiting times for these groups. Among registrants with a low PRA (0-9\%), African Americans had a median time to transplant more than twice that of whites, and Hispanic/Latino patients had a median time to transplant that was 1.5 times that of whites.

The death rate on the waiting list for children aged 11-17 years was about half that of children aged 1-10 years. For adults, the death rate on the waiting list rises with increasing age. However, the death rates for patients under age 50 actually have decreased over the past 10 years. The death rate for patients aged 65 years and older is approximately four times the rate for those aged 18-34 years.

\section{Kidney transplant recipient characteristics}

The number of kidney-alone transplants increased by $6 \%$ between 2003 and 2004, from 14856 to 15 671, after an average annual increase of approximately $4 \%$ between 1995 and 2003 . There was a $43 \%$ increase in transplants between 1995 and 2004, with the biggest numerical increase occurring in the number of living donor transplants, which doubled during the decade. Deceased donor transplants increased 19\% over this same time period. In 2004 the number of deceased donor transplants increased by
$8 \%$ over 2003 , while the number of living donor transplants increased by only $3 \%$. This represents a reversal of previous trends. Deceased donor transplants represented 54\% of kidney-alone transplants performed in 2004, which was comparable to the percentage in 2003.

Donor source: The overall number of donors in the United States continues to increase. The number of living kidney donors once again exceeded the number of deceased kidney donors, continuing a trend that began in 2000. However, the number of deceased donor kidney transplants increased by $8 \%$, from 8388 in 2003 to 9025 in 2004, by far the largest increase in a decade. The annual rate of increase since 1995 averaged $2 \%$, and the average annual rate of increase since 1999 was 3\%. Numerically, most of this increase was in standard criteria donor (SCD) transplants, which increased by 7\% compared with 2003 (Figure 1). Initiatives at the national level, such as the Organ Donation Breakthrough Collaborative, as well as activities at the local level are likely to contribute to the increasing rate of donation among deceased donors. The number of kidneys transplanted from living donors increased by only $3 \%$ from 2003 to 2004, but since 1995 the annual rate of increase averaged $8 \%$.

In analyses of deceased donor kidney transplants, the SRTR distinguishes between expanded criteria donor (ECD) transplants and non-expanded criteria donor (nonECD) transplants. An expanded criteria donor has general health or organ-specific characteristics that preclude him or her from being considered an 'ideal' donor. However, the inclusion of such donors in the donor pool provides transplantation to those who might not be able to wait for an ideal donor. The non-ECD category, as defined in the SRTR analyses, includes transplants of both SCD kidneys (meaning kidneys from donors who have experienced brain death) and DCD kidneys (meaning kidneys from donors whose hearts have irreversibly stopped beating). Transplants from DCD donors who meet the ECD criteria (DCD/ECD) are counted as ECD transplants.

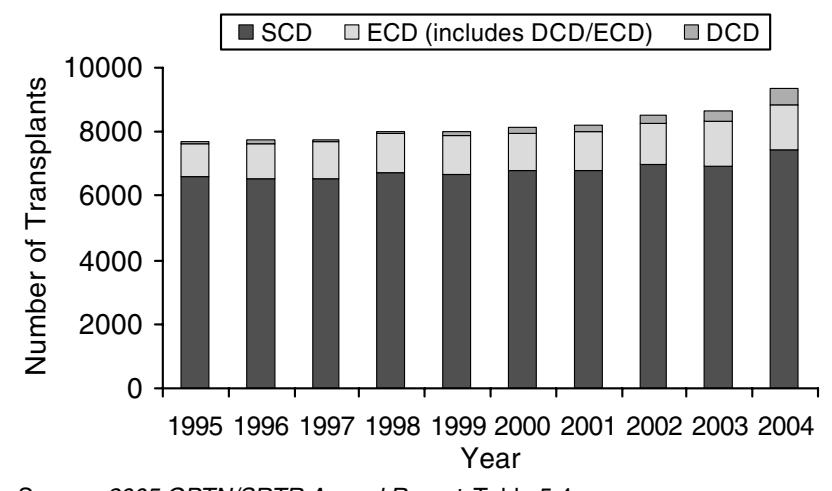

Source: 2005 OPTN/SRTR Annual Report, Table 5.4

Figure 1: SCD, ECD and DCD kidney transplants, 1995-2004. 
Although kidney allocation algorithms based on the definition of ECD were implemented on October 31, 2002, the ECD criteria were applied to donors prior to that time for the purpose of analyzing trends. In 1995 ECD kidneys accounted for $13 \%$ of the kidneys transplanted from deceased donors. In 2004, ECD kidneys accounted for $15 \%$ of the kidneys transplanted from deceased donors (1439 kidneys). From 1995 to 2004, the number of ECD kidney transplants increased at an average annual rate of $4 \%$, while SCD kidney transplants increased at a rate of only 1\% per year. However, between 1999 and 2004, transplants of kidneys from both groups increased at similar average annual rates of $2.2 \%$ for SCD and $2.5 \%$ for ECD.

Donation after cardiac death has increased steadily since 2000. Although the absolute numbers remain small, the number of kidneys transplanted from DCD donors increased by 39\% between 2003 and 2004. Between 1999 and 2004, the annual increase in DCD kidney transplants averaged $30 \%$. Sixty-one kidneys were transplanted in 2004 from DCD donors who also met criteria of ECD donors. This represents $0.7 \%$ (or 1 in 150) of all deceased donor kidney transplants.

There has been a steady increase in the allocation of kidneys from deceased donors to recipients of other organs. In 2004, 328 kidneys from deceased donors went to recipients of multi-organ transplants, not including KP transplants. This number approximately doubled between 2000 and 2004, and represented 4\% of all deceased donor kidneys transplanted in 2004. The recent increase in multi-organ kidney transplants is largely due to an increase in combined liver-kidney transplants and may be related to the implementation of the Model for End-Stage Liver Disease (MELD) algorithm in February 2002. The MELD scoring system awards points for diminished renal function, thereby favoring those liver transplant candidates who may also require a renal transplant (Figure 2).

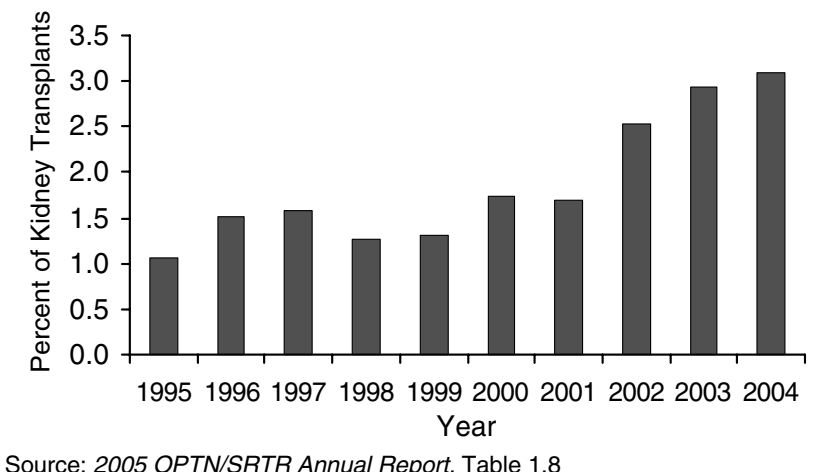

Source: 2005 OPTN/SRTR Annual Report, Table 1.8

Figure 2: Non-SPK multiple organ transplants as \% of total deceased donor kidney transplants, 1995-2004.

\section{Characteristics of deceased donor non-ECD kidney recipients}

Age: The trend toward an increasing number of kidney transplants among patients aged 65 years and older continued in 2004. In this age group, the absolute number of deceased donor non-ECD kidney transplants (that is, transplants from SCD and DCD donors) has more than doubled since 1995, reaching 849 transplants in 2004. This number represents $11 \%$ of all deceased donor kidney transplants, up from $6 \%$ in 1995. The number of 50-64 year-old kidney transplant recipients increased by 49\%, from 2028 in 1995 to 3020 in 2004 . Recipients in this age group accounted for $38 \%$ of deceased donor kidney transplants, up from $30 \%$ in 1995. Remarkably, both the absolute number and the proportion of deceased donor kidney transplants performed in individuals aged 18-49 years decreased, from 3943 (59\% of all deceased donor non-ECD kidneys transplanted) in 1995 to 3668 (46\% of all deceased donor non-ECD kidneys transplanted) in 2004. For recipients aged 0-17 years, there was a minimal increase in the number (322 in 1995 to 378 in 2004) and no change in the proportion (4.8\% in 1995 to $4.7 \%$ in 2004) of deceased donor non-ECD kidney transplants.

From 1995 to 2004 the proportion of recipients of deceased donor non-ECD kidney transplants who had received a prior kidney or KP transplant was unchanged at about 15\%. Sixty percent of the recipients of deceased donor non-ECD kidney transplants were male, and this also was unchanged since 1995.

Race: In 1995, deceased donor non-ECD kidney recipients were 58\% white, 27\% African American, 11\% Hispanic/Latino and $4 \%$ Asian. Beginning in 1996, there was a steady decrease in the proportion of white deceased donor non-ECD kidney recipients and a corresponding increase in the percentage of African Americans, Hispanics/Latinos and Asians. However, in 2004, as in 2003, $49 \%$ of deceased donor non-ECD recipients were white, $30 \%$ were African American, 14\% were Hispanic/Latino and 6\% were Asian. Although the change in allocation policy enacted in May 2003, which decreased overall points for HLA matching and instead favored waiting time, has not yet shown obvious impact on the racial distribution of transplant recipients, a recent study comparing racial distributions pre- and post policy implementation demonstrated an increase in the proportion of transplants allocated to minorities (SRTR special analysis, September 2005).

Payer source: In 2004, Medicare was the primary payment source for recipients of deceased donor nonECD kidney transplants, covering $62 \%$ of the transplants. Together, Medicare and Medicaid provided primary coverage for $67 \%$ of kidney transplants. Private insurance covered $30 \%$ of the deceased donor transplants. These proportions are unchanged since 1995. 
Primary diagnosis: The distribution of the primary diagnosis for ESRD changed little between 1995 and 2004 for deceased donor non-ECD kidney recipients. Glomerular diseases accounted for about $25 \%$, diabetes and hypertensive nephrosclerosis for about $20 \%$ each, and PKD for $8 \%$ of the transplant cases.

HLA mismatch distribution and prior sensitization: In 2004, there were more HLA zero-mismatched deceased donor non-ECD kidney transplants than ever before: the 1343 recipients of zero-mismatched deceased donor kidneys represented $17 \%$ of the deceased donor non-ECD transplants. However, since 1995 there has been a steady decrease in the number and proportion of non-ECD transplants with one, two or three HLA antigen mismatches. In 1995, these transplants represented $38 \%$ of the deceased donor non-ECD transplants, while in 2004 this group accounted for only $17 \%$ of the deceased donor non-ECD transplants. Transplants into recipients with four, five or six HLA antigen mismatches in 2004 represented nearly two thirds of all the deceased donor non-ECD transplants (26\%, 28\% and $11 \%$, respectively).

Similar trends are noted for HLA-DR mismatches. Zero DR mismatches decreased from 35\% in 1995 to $28 \%$ in 2004, with single DR mismatches remaining unchanged at $43 \%$ and two DR mismatches increasing from $21 \%$ in 1995 to $28 \%$ in 2004 .

There was continued improvement in transplantation for individuals with high PRA. From 1995 to 2004 the number of deceased donor non-ECD kidney transplants into recipients with a PRA of $80 \%$ or higher at the time of transplant more than doubled (390 in 2004), and the proportion increased from $2.8 \%$ to $4.9 \%$. In 2004 there were 1140 deceased donor non-ECD transplants into recipients with a PRA at transplant of $10-79 \%$, representing $14 \%$ of transplants in this group, up from $12 \%$ in 1995 . Non-ECD transplants into recipients with a peak PRA of $80 \%$ or higher nearly doubled between 1995 and 2004. The proportion increased from $8 \%$ to $13 \%$, while the proportion transplanted into recipients with a peak PRA of $10-79 \%$ decreased from $27 \%$ to $22 \%$

Cold ischemia time: In 2004, $18 \%$ of all deceased donor non-ECD kidney transplants were completed with cold ischemia times (CITs) of $11 \mathrm{~h}$ or less, $40 \%$ with CITs of 12 21 h, $22 \%$ with CITs of $22-31$ h, and $4 \%$ with CITs of 32$41 \mathrm{~h}$. This represents a $7 \%$ increase over 1995 in the proportion of transplants performed with CITs less than $12 \mathrm{~h}$, and a $12 \%$ decrease in the proportion of transplants with CITs of $22-31 \mathrm{~h}$. Only $0.5 \%$ of non-ECD transplants were performed with CITs of $42 \mathrm{~h}$ or more. These overall percentages have changed little since 2000.

\section{Characteristics of ECD kidney recipients}

The age distribution of ECD kidney recipients was different than that of non-ECD kidney recipients. In 2004, only three ECD kidneys were transplanted into recipients less than 18 years of age $(0.2 \%)$. Seventy-eight percent of ECD kidney recipients were more than 50 years old (compared to $49 \%$ of non-ECD kidney recipients). ECD kidneys were less likely than non-ECD kidneys to go to recipients of repeat kidney transplants $18.5 \%$ of ECD kidneys were used in repeat transplants compared to $14.5 \%$ of non-ECD kidneys).

There was a steady decline in the percentage of zeroantigen mismatched ECD kidney transplants, from 13\% of the total ECD kidneys transplanted in 1995 to only $5 \%$ in 2004. While zero- and one-antigen mismatches were less frequent among ECD recipients than among nonECD recipients, two-, three- and four-antigen mismatches were approximately as common among ECD recipients as among non-ECD recipients. Five- and six-antigen mismatches were more common among ECD recipients than among non-ECD recipients, representing 35\% and 18\%, respectively, of all the ECD kidney transplants performed in 2004. These proportions are more than twice those observed in 1995 for five- and six-antigen mismatches in ECD recipients. Thus, this older group of patients is receiving kidneys with a poorer HLA match.

On the other hand, sensitized recipients were less likely to receive an ECD kidney. Only $1.5 \%$ of ECD recipients had a PRA of $80 \%$ or higher, and only $10 \%$ had a PRA of $10-79 \%$ (compared to $5 \%$ and $14 \%$ among non-ECD recipients).

In 2004, ECD kidney recipients were less likely than the recipients of non-ECD kidneys to have glomerular disease (17\% vs. $26 \%$ ) and more likely to have diabetes (32\% vs. $22 \%$ ) or hypertension (24\% vs. $20 \%$ ). The distribution of CITs for ECD transplant recipients is the same as the distribution for non-ECD recipients, with a CIT $\leq 31 \mathrm{~h}$ for approximately $80 \%$ of non-ECD and ECD transplants, and $\leq$ $21 \mathrm{~h}$ for $58 \%$ of non-ECD and $56 \%$ of ECD transplants. However, CITs have improved modestly for recipients of ECD kidneys over the past decade: In 2004, 16\% were transplanted with less than $12 \mathrm{~h} \mathrm{CIT,} \mathrm{compared} \mathrm{with} \mathrm{only}$ $7 \%$ in 1995 and $11 \%$ in 2001 . Similarly, in 2004 , 56\% of ECD kidneys had CITs of less than $22 \mathrm{~h}$, compared with $50 \%$ in 1995 . Unfortunately, there has been little change since 2002 in the overall CIT distribution for recipients of ECD kidneys.

\section{Characteristics of living donor kidney recipients}

Compared to the non-ECD kidney recipients, living donor recipients demonstrated the same trends in age distribution and the same gender distribution. In 2004 proportionately more recipients of living donor kidneys than non-ECD recipients were white (64\% vs. 49\%), and fewer were African American (14\% vs. $30 \%$ ) or Asian (4\% vs. $6 \%$ ). Fewer living donor recipients had had prior transplants (12\% vs. $16 \%$ for non-ECD recipients). 
The payment source for living donor kidney recipients in 2004 was different than that for non-ECD recipients. Private insurance is the primary payment source for $57 \%$ of living donor kidney transplant recipients, Medicare for $35 \%$ and Medicaid for 5\% (compared to 30\%, 62\% and 6\%, respectively, for non-ECD kidney recipients).

The relationship of the living donor to the recipient changed dramatically between 1995 and 2004. The proportion of donors who were parents of recipients decreased from $22 \%$ in 1995 to $11 \%$ in 2004 , and donation from a sibling decreased from $45 \%$ to $28 \%$ during the same period. The proportion of kidneys donated by the adult child of a recipient was unchanged, the proportion donated by a spouse increased from $8 \%$ to $12 \%$, and the proportion donated by 'other unrelated' individuals, such as friends, increased from 5\% to $21 \%$ between 1995 and 2004.

The marked change in the living donor relationship has had an impact on HLA matching. In 2004 living donors with zero antigen mismatches accounted for $9 \%$ of the transplants; one mismatch, 6\%; two mismatches, 18\%; three mismatches, 29\%; four mismatches, $13 \%$; five mismatches, $16 \%$ and six mismatches, 8\%. In 1995 living donors with zero antigen mismatches accounted for $18 \%$ of the transplants; five mismatches, 7\%; and six mismatches, 3\%. Similar trends occurred in HLA-DR matching. Living donor kidney recipients remained less sensitized on average than non-ECD recipients, with only $2 \%$ of the living donor recipients having a PRA of $80 \%$ or higher at transplant. Ten percent had a PRA of $10-79 \%$ at transplant. This compares to $5 \%$ with a PRA of $80 \%$ or higher and $14 \%$ with a PRA of $10-79 \%$ among recipients of deceased donor non-ECD kidneys in 2004.

Preemptive kidney transplantation has been demonstrated to provide better outcomes than transplantation after any period of time on dialysis (5). The number of preemptive transplants more than doubled over the past decade, and the percentage of preemptive transplants increased from $9 \%$ to $13 \%$ during this period (SRTR special analysis, July 2005). Virtually all of this increase was in living donor transplants; $24 \%$ of all living donor transplants were preemptive in 2004 (compared with 18\% in 1995). As expected, only $4.3 \%$ of deceased donor transplants were preemptive, a minimal increase from $4.1 \%$ in 1995.

\section{Immunosuppression and acute rejection}

Induction immunosuppression was used for $72 \%$ of kidney recipients in 2004, compared to $27 \%$ of recipients in 1995. Rabbit antithymocyte globulin (ATG) (Thymoglobulin ${ }^{\circledR}$, SangStat Medical Corp., Fremont, CA), which was used in 37\% of transplants in 2004 and $0 \%$ in 1995, has replaced muromonab-CD3 (Orthoclone OKT3 ${ }^{\circledR}$, Orthobiotech, Bridgewater, NJ), which was used in $25 \%$ of transplants in 1995 and $0.3 \%$ in 2004, as the most common induction agent. Anti-Tac antibodies were used in 30\% and alemtuzumab (Campath-1 $\mathrm{H}^{\circledR}$, ILEX Pharmaceuticals, San Antonio, TX) in 7\% of kidney transplants in 2004.

Steroid use recorded at discharge from the transplant hospital decreased from $94 \%$ of the recipients in 2001 to $78 \%$ in 2004 . Tacrolimus was used at discharge for $72 \%$ of the recipients in 2004 and cyclosporine for $21 \%$. Eighty-four percent of the recipients were on an anti-metabolite, which was mycophenolate in $81 \%$ of those recipients. Twelve percent of the recipients were on sirolimus at discharge, and $21 \%$ received sirolimus during the first year.

There has been significant progress in the prevention of acute rejection in the first year after transplantation. In 1994, 32\% of kidney transplant recipients required treatment for acute rejection, and by 1996 the percentage had risen to $51 \%$. Remarkably only $13 \%$ of recipients of transplants in 2003 were treated for rejection in the first year after transplant, despite the reduction in steroid use and the decrease on average in HLA matching.

\section{Kidney transplant graft survival}

In the 2005 OPTN/SRTR Annual Report, graft survival is reported with 1-year survival representing the cohort of transplants performed during 2002-2003, 3-year survival representing the cohort of transplants performed in 2000-2003, and 5-year survival representing the cohort of transplants performed in 1998-2003. Overall adjusted graft survival rates for recipients of deceased donor non-ECD kidneys were $91 \%$ at 1 year and $69 \%$ at 5 years (Figure 3). Adjusted survival analyses allow for comparison of survival rates holding 'all else equal'; for instance, a survival rate reported by diagnosis and adjusted for sex, race, and age allows the reader to compare the rate for diabetes to the rate for glomerular diseases as if the patient mix is the same in both diagnosis groups. Unadjusted survival analyses produce overall rates for a given group; differences in these rates across groups may be affected by differences in the case mixes of the groups.

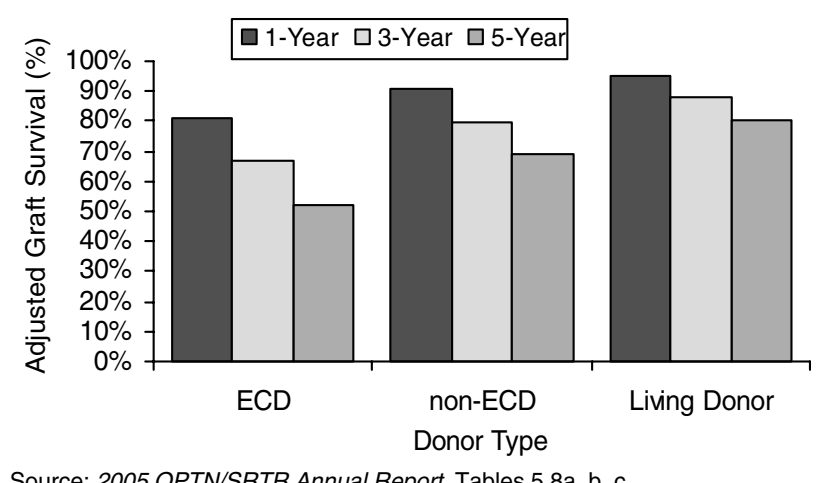

Source: 2005 OPTN/SRTR Annual Report, Tables 5.8a, b, c.

Figure 3: Adjusted 1-year, 3-year and 5-year kidney graft survival, by donor type, for transplants received 1998-2003. 
Comparative graft survival rates for recipients of ECD kidneys were significantly lower than those for living donors; $81 \%$ at 1 year $(p<0.05)$ and $53 \%$ at 5 years $(p<0.05)$ after transplantation. Among living donor kidney recipients, the 1-year graft survival rate was $97 \%$ and the 5-year graft survival rate was $80 \%$. Kidneys transplanted from deceased donors, especially those considered expanded criteria donors, were associated with greater long-term attrition than were kidneys transplanted from living donors.

Graft survival among deceased donor non-ECD kidney recipients: Graft survival rates among non-ECD kidney recipients (that is, those with kidneys from SCD or DCD donors) differed little by recipient sex or transplant center volume.

One-year non-ECD graft survival was best in patients aged 11-64 (90-92\%) and slightly lower in adults aged 65 years and older (88\%). At 5 years after transplantation, kidney survival rates were best among recipients aged 35-49 years $(73 \%)$ and worst among recipients aged 65 years and older (63\%). There were minimal differences between racial and ethnic groups in graft outcomes during the first posttransplant year. Beyond this time, African Americans continued to experience the worst outcomes-60\% graft survival at 5 years. Asians enjoyed the best 5 -year graft survival rate-78\%. As has been reported previously, 1- and 5-year deceased donor non-ECD kidney survival rates are superior in patients with PKD $(95 \%$ and $79 \%)$; in distinct contrast, 5-year graft survival is $14-15 \%$ lower for patients with diabetes, hypertensive nephrosclerosis and vascular diseases. Surprisingly, over the past decade, 1-year, 3-year and 5-year unadjusted deceased donor non-ECD graft survival rates have improved only $2-3 \%$.

Posttransplant outcomes continue to vary by donor age. Kidneys from the very young and, in particular, kidneys from older donors fare the worst (Figure 4). The effects of CIT on non-ECD graft survival rates at 1 year were minimal $(92 \%$

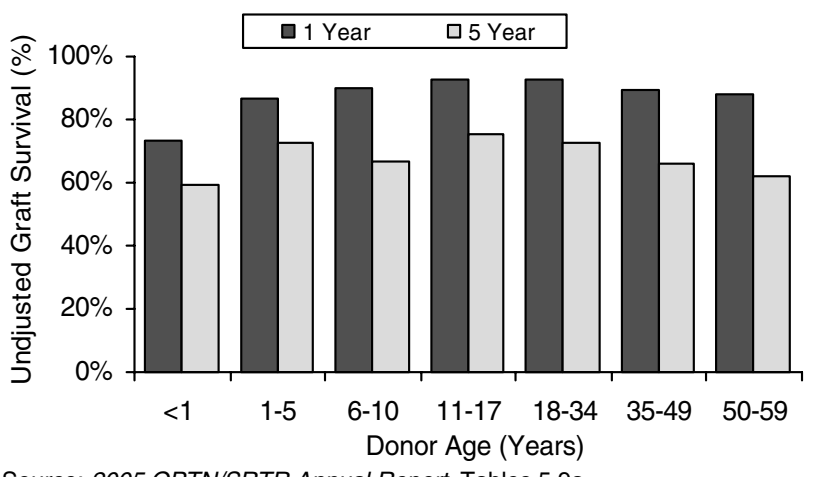

Source: 2005 OPTN/SRTR Annual Report, Tables 5.9a.

Figure 4: Unadjusted 1-year and 5-year deceased donor nonECD kidney graft survival, by donor age, for transplants received 1998-2003.
Table 1: Unadjusted graft survival rates at 5 years after kidney transplantation, by degree of HLA mismatch and donor type, for transplants received in 1998-2003

\begin{tabular}{llll}
\hline \multirow{2}{*}{$\begin{array}{l}\text { Level of } \\
\text { HLA mismatch }\end{array}$} & \multicolumn{3}{l}{ Survival rate (\%) by donor type } \\
\cline { 2 - 4 } & ECD (\%) & non-ECD (\%) & Living (\%) \\
\hline 0 & 60 & 74 & 87 \\
1 & 53 & 71 & 80 \\
2 & 57 & 71 & 80 \\
3 & 52 & 70 & 79 \\
4 & 52 & 69 & 79 \\
5 & 50 & 66 & 81 \\
6 & 47 & 65 & 79 \\
\hline
\end{tabular}

Source: 2005 OPTN/SRTR Annual Report, Table 5.9.

for CITs of $0-11 \mathrm{~h}$ compared to $91 \%$ for CITs of $32-41$ h), although by 5 years after transplantation, graft survival was $72 \%$ for $\mathrm{CITs}$ of $0-11 \mathrm{~h}$ and $65 \%$ for CITs of $32-41 \mathrm{~h}$. It is not surprising that those requiring dialysis within the first posttransplant week have worse 5-year graft survival; in the 1998-2003 cohort of transplants, the graft survival rate for non-ECD kidneys was $54 \%$ if dialysis was needed in the first week and $74 \%$ if dialysis was not needed.

No differences are seen in non-ECD kidney 1-year graft survival by recipient PRA, although outcomes at 5 years after transplantation declined with increasing levels of sensitization: $70 \%$ survival rate for PRAs of 0-9\%, 63-65\% survival rate for PRAs above $9 \%$ and $63 \%$ for very highly sensitized recipients (PRA > 80\%). As shown in Table 1, 5-year non-ECD graft survival outcomes differed by level of HLA mismatch, at 74\% for zero-mismatched transplants and $65 \%$ for transplants with a six-antigen mismatch. For recipients of ECD kidneys, 5-year graft survival rates were $60 \%$ for zero-mismatched and $47 \%$ for six-antigen mismatched transplants. Both non-ECD and ECD recipients with intermediate degrees of mismatching had intermediate graft survival rates.

Graft survival among ECD kidney recipients: Among ECD kidney recipients, unadjusted 1-year graft survival ranged between $79 \%$ and $83 \%$ for all ages. By 5 years after transplant, graft survival was $57 \%$ for those aged 35 49 years, $54 \%$ for those aged $50-64$ years, and only $44 \%$ in the oldest patients. African Americans continued to experience the worst overall ECD graft survival rates-only $43 \%$ at 5 years. In contrast, as with non-ECD kidneys, Asians enjoyed the best 5 -year graft survival rates at $65 \%$ for ECD kidneys. One- and 5-year ECD kidney survival rates are superior in patients with PKD (87\% and 67\%, respectively). Over the past decade, there has been only a very modest (3-5\%) increase in 1-, 3- and 5-year ECD kidney graft survival rates.

There were modest differences in 1-year graft survival for ECD kidneys by recipient PRA $181 \%$ for those with PRAs of $0-9 \%$ and $75-77 \%$ for those with PRAs of $10+\%$ ). Outcomes at 5 years after transplantation were best for 
those with low PRAs: 54\% for those with PRAs of 0-9\%, $43 \%$ for those with intermediate levels of sensitization, and $44 \%$ for very highly sensitized recipients (PRA $\geq 80 \%$ ). As shown in Table 1, among recipients of ECD kidneys, the 5 -year graft survival rate was $60 \%$ for zero-mismatched transplants and $47 \%$ for six-antigen mismatched transplants. Like recipients of non-ECD kidneys, recipients with intermediate degrees of mismatching had intermediate graft survival rates. The fact that recipients of a zeromismatched ECD kidney fared worse than those with a zero-mismatch non-ECD allograft, along with the superior results of living donor transplantation for any degree of HLA mismatch, highlights the overriding influence of organ quality on allograft outcome, independent of immune-mediated injury.

Notably, for recipients of ECD kidneys, 1-year and 5-year graft survival rates were $84 \%$ and $58 \%$ for $\mathrm{ClTs}$ of 0 $11 \mathrm{~h}$, and $75 \%$ and $46 \%$ for CITs of $32-41 \mathrm{~h}$. Approximately $35 \%$ of ECD kidney recipients, compared to $22 \%$ of non-ECD recipients, required dialysis in the first posttransplant week. In addition, the requirement for dialysis within the first posttransplant week continues to be associated with adverse 5-year ECD kidney graft survival rates, as it does for non-ECD kidneys. The comparative rates for ECD transplant recipients were $41 \%$ in patients who required dialysis and $59 \%$ for those with immediate graft function. ECD kidney recipients with previous transplants experienced worse outcomes at all times following transplant compared with those who received a primary transplant. Survival rates were $73 \%$ at 1 year, $57 \%$ at 3 years, and $46 \%$ at 5 years among recipients with previous transplants, compared to $82 \%, 68 \%$ and $53 \%$ among primary transplant recipients. Overall, the generally inferior graft outcomes observed with ECD kidneys underscore the negative effects of suboptimal graft quality.

\section{Graft survival among living donor kidney recipients:} Among recipients of living donor kidneys, 1-year graft survival ranged from $93 \%$ among patients aged 65 years and older to $96 \%$ among recipients in the $1-5$ year-old age group. For 5-year graft survival, patients 1-10 years old fared best (90-95\% survival), while those aged 65 years and older had the worst outcomes at $70 \%$. Graft survival rates among recipients of living donor transplants varied little by demographic factors and did not differ by recipient gender. Five-year graft survival rates were 4\% lower for those with prior transplantation, a traditional risk factor for inferior outcomes. While 1-year graft survival rates were minimally different by ethnicity (3\% difference between the ethnic groups with the best and worst outcomes), 5year survival was clearly best among Asians (84\%) and was $12 \%$ lower among African Americans (72\%). As observed among deceased donor recipients, the best 5-year graft survival rates were found among recipients whose cause of ESRD or chronic kidney disease was secondary to PKD.
The worst outcomes were noted in patients with diabetes, hypertensive nephrosclerosis and vascular disease.

From an immunological perspective, graft survival at 1 year was $5 \%$ lower in patients with a PRA of $80 \%$ or higher, while there was no difference with lower levels of sensitization (94-95\% for PRAs of 0-79\%). By 5 years after transplantation, patients with a PRA of 0-9\% enjoyed $80 \%$ graft survival, and this progressively declined to $74 \%$ for patients with PRAs of $80 \%$ or more. There was only a $3 \%$ difference in 1-year graft survival between zero-mismatched and six-antigen mismatched transplants. However, by the end of the fifth posttransplant year, while graft survival was $87 \%$ for zero-mismatched transplants, it was consistently 7-8\% lower for all other degrees of HLA mismatching. Finally, although transplants from siblings consistently had the highest graft survival rates, the difference at 5 years after transplantation was particularly striking (84\% survival rate for sibling transplants compared to a high of $80 \%$ for any other group). This likely represents the contribution of two-haplotype match transplants to this pool of donors.

Dialysis within the first posttransplant week is a harbinger of a particularly poor graft outcome for living donor kidney recipients, as it is for deceased donor kidney recipients. The 1-year graft survival rate was markedly diminished for recipients who required early posttransplant dialysis (65\%) compared to those who did not (97\%). Interestingly, it was even lower than comparative survival rates for recipients of ECD and non-ECD kidneys. The 32\% difference in 1-year graft survival described above was maintained at 5 years after transplantation. It is likely that the need for dialysis early after a living donor transplant is due to major technical complications resulting in early kidney loss, rather than acute tubular necrosis (ATN), which is the cause of delayed graft function (DGF) in the vast majority of deceased donor transplants and has a very high rate of recovery. Kidneys from donors 65 years of age and older had the lowest graft survival rates at $87 \%$ at 1 year and $73 \%$ at 5 years. For all other donor age groups, graft survival ranged between $94 \%$ and $96 \%$ at 1 year and between $79 \%$ and $82 \%$ at 5 years after transplantation. In contrast to deceased donor organs, living donor kidneys are accepted only after rigorous evaluation and screening of potential donors, including detailed assessment of their renal function. In addition, when living donor organs are transplanted, CIT is minimized and the transplant is performed under optimal conditions.

\section{Kidney transplant recipient survival}

Adjusted patient survival curves for recipients of living and deceased donor kidneys are shown in Figure 5. One-year patient survival ranged from $91 \%$ for ECD kidney recipients to $98 \%$ for patients receiving kidneys from living donors. By 5 years after transplantation, patient survival was $20 \%$ lower among recipients of ECD 


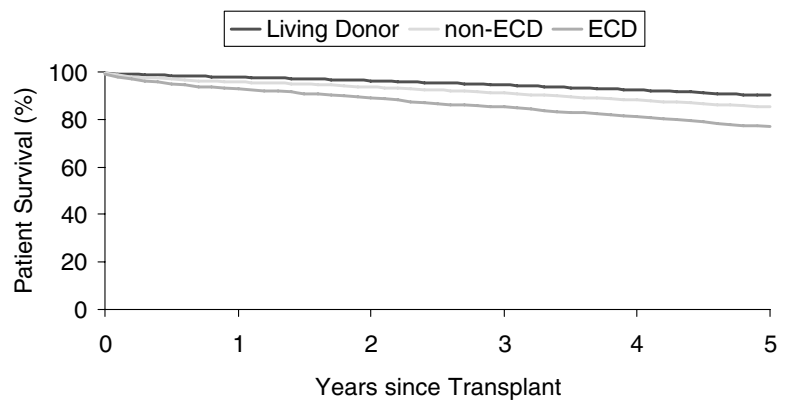

Source: SRTR Analysis, May, 2005.

Figure 5: Patient survival for kidney transplant recipients, 1998-1999.

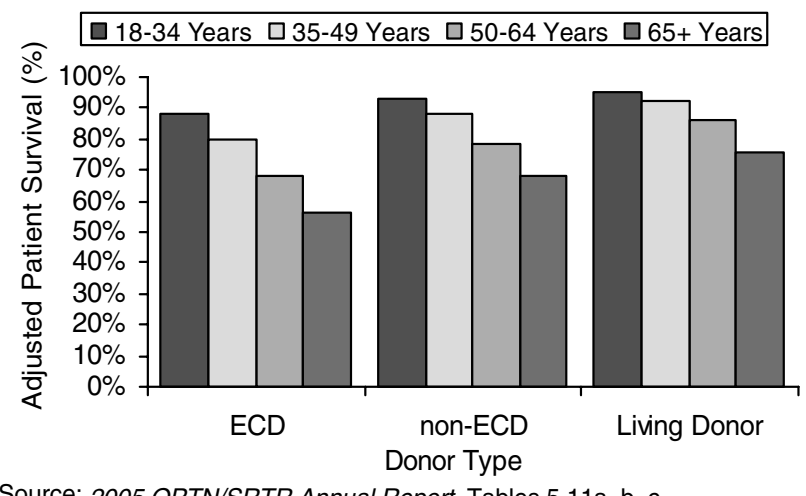

Source: 2005 OPTN/SRTR Annual Report, Tables 5.11a, b, c.

Figure 6: Adjusted 5-year patient survival for kidney recipients, by age and donor type, for transplants received 19982003.

kidneys (70\%) than among living donor kidney recipients (90\%) and $15 \%$ less than among deceased donor non-ECD kidney recipients (85\%). This discrepancy likely reflects the trade-off of trying to expeditiously transplant organs to candidates who are older and/or have increased comorbidities by using a greater proportion of inferior-quality deceased donor kidneys. As shown in Figure 6, this trade-off is underscored by the fact that among recipients of ECD kidneys, 5-year survival rates were poorest among recipients who were 65 years old or older at the time of transplant (56\%). Those with diabetes as the cause of kidney failure have a similarly poor 5year survival rate of $60 \%$. Among individuals who received transplants from a living donor, those aged 65 years and older had a 5 -year survival rate of $76 \%$ and those with diabetes had a survival rate of $86 \%$. Among deceased donor non-ECD kidney recipients, those aged 65 years and older had a survival rate of $68 \%$ and those with diabetes had a survival rate of $78 \%$. Moreover, while 5-year patient survival rates remained stable over the past 5 years for living and deceased donor non-ECD kidney recipients, they actu- ally declined by $2 \%$ over that time period among patients who received an ECD kidney.

Since 1995, 1-year death rates for deceased donor nonECD kidney recipients aged 18-64 years have declined by 20-25\%. The most dramatic change was seen in recipients aged 65 years or older, whose death rate decreased from 164 to 78 deaths per 1000 patient-years at risk. Similar improvements occurred with living kidney donor recipients. However, while improvements in 1-year death rates are observed in most age groups, death rates among ECD kidney recipients aged 65 years and older actually increased from 112 to 153 deaths per 1000 patient-years at risk. Death rates were higher for whites and African Americans than for Hispanics or Asians.

Only slight differences were seen in patient survival at 1 and 3 years among recipient ethnicities, regardless of whether the transplant was from a living donor, an ECD deceased donor or a non-ECD deceased donor. On the other hand, 5-year survival rates were associated with recipient ethnicity. Asian and Hispanic/Latino recipients consistently had higher 5-year patient survival rates across all classes of kidney donors, generally 2-11\% higher than patient survival rates among whites and African Americans.

Five-year adjusted patient survival varied by primary cause of ESRD. Those with PKD and glomerulonephritis consistently experienced better survival rates than those with diabetes. Unadjusted patient survival at all times after transplantation was lower for those who required dialysis in the first week after transplant in all donor categories, perhaps paralleling reduced allograft survival. The effect was most notable among recipients of living donor kidneys. In this group, patients requiring dialysis within the first week after transplantation experienced a 78\% 5-year survival rate, whereas those with immediate graft function experienced a $91 \%$ survival rate.

There were minimal differences in 5-year patient survival rates by HLA mismatching in any donor category, except at the extremes of mismatching (Table 1). Zero-mismatched kidneys experienced enhanced survival when compared to six-antigen mismatched transplants in living donor recipients (93\% survival for zero-mismatched kidneys vs. $90 \%$ for six-antigen mismatched kidneys), ECD kidney recipients (73\% survival for zero-mismatched kidneys vs. $66 \%$ for six-antigen mismatched kidneys) and deceased donor non-ECD kidney recipients $(84 \%$ survival for zeromismatched kidneys vs. $81 \%$ for six-antigen mismatched kidneys).

\section{Prevalence of people living with a functioning kidney transplant}

Along with the increase in the number of transplants performed, there has been an increase in the number of people living with a functioning renal allograft. In fact, the 
number of people living with a functioning kidney transplant doubled between 1995 and 2004. At the end of 1995 there were 50529 people living with a functioning kidney transplant and 3156 people living with a functioning KP transplant. At the end of 2004 there were 101440 people living with a functioning kidney transplant and 7213 people living with a functioning KP transplant. Based on U.S. Census figures for the same years, this represents 3.4 people per 10000 living with a functioning kidney transplant and 3.7 people per 10000 living with a kidney or KP transplant in 2004, up from 1.9 and 2.1 per 10000 in $1995(3,6)$. Health care providers in all settings are increasingly likely to be exposed to these organ transplant recipients. Based on data from the 2004 annual report of the U.S. Renal Data System, which provides prevalence data through 2002, kidney and KP recipients living with a functioning kidney transplant represented $18 \%$ of all ESRD patients in 1995 and $21 \%$ of all ESRD patients in 2002 (2).

Among people living with a functioning kidney-only transplant at the end of 2004, the proportions of children (aged 0-10 years), adolescents (aged 11-17 years) and early middle aged adults (age 35-49 years) have changed little compared to 1995 , but the proportion of young adults (aged 18-34 years) decreased from 28\% in 1995 to $20 \%$ in 2004. At the same time, the proportion of people living with a functioning kidney transplant in the 50-64 age group increased from $25 \%$ to $32 \%$ (a $30 \%$ increase in proportion) and the proportion of people living with a functioning kidney transplant in the 65 and older age group increased from $3.8 \%$ to $7.4 \%$ (a $94 \%$ increase in proportion).

Compared to 1995, slightly fewer people with a functioning kidney transplant in 2004 were white (62\% in 2004 vs. 66\% in 1995) and slightly more were African American (19.9\% in 2004 vs. $19.2 \%$ in 1995), Hispanic/Latino (12\% vs. 10\%) or Asian (5\% vs. 4\%).

Slightly more people living with a functioning kidney transplant in 2004 than in 1995 received a zero-mismatched organ (14\% in 2004 vs. $12 \%$ in 1995). Slightly fewer received a zero or one HLA-DR mismatched kidney (30\% and $47 \%$ in 2004 vs. $33 \%$ and $49 \%$ in 1995), and slightly more received a doubly HLA-DR mismatched organ (22\% in 2004 vs. $16 \%$ in 1995).

Thirty percent of people living with a functioning kidney transplant in 2004 received a transplant for a diagnosis of glomerular disease, $19 \%$ for diabetes, $14 \%$ for hypertensive nephrosclerosis, 10\% for PKD, 7\% for tubular or interstitial disorders and $0.3 \%$ for neoplastic disease. This distribution has not changed over the past decade.

\section{Simultaneous Pancreas-Kidney Transplantation}

\section{The SPK waiting list}

The waiting list for an SPK transplant decreased from a peak of 2062 active candidates at the end of 2000 to 1720 active candidates at the end of 2004 . This decrease can be attributed to an overall decline in the number of new registrants on the waiting list from 2000 to 2004, as the number of SPK transplants also decreased. While this decline may reflect uncertainty about whether SPK offers a patient and graft survival advantage over kidney transplant alone in type 1 diabetics $(7,8)$, it may also be a consequence of the increase in living donor kidney transplants and subsequent pancreas after kidney (PAK) registrations over this time period. This trend may be changing, as SPK registrations increased from 1649 in 2003 to 1735 in 2004. The percentages of African American registrants (18\%) and Hispanic/Latino registrants $(11 \%)$ increased over the past decade. The age distribution of candidates on the waiting list remains shifted toward older candidates. In 2004, 20\% of candidates were aged 18-34 years, $61 \%$ were aged 3549 years and $18 \%$ were aged $50-64$ years; this distribution is similar to the age distribution in 2003. The absolute number of active waiting list patients aged 18-34 years declined to roughly the same number that were on the waiting list in 1995. The decline might reflect a preference for pursuing living donor kidney transplants for younger patients, rather than placing the patients on the waiting list for SPK. Less than $0.5 \%$ of candidates were either age 65 or older or younger than 18 years. Fourteen percent of SPK candidates had received a previous transplant, and $5 \%$ had received a previous SPK transplant.

Overall waiting times continued to increase as of the end of 2004 , with $44 \%$ of the active waitlisted patients having waited for more than a year. This is an increase from $25 \%$ at the end of 1995 and $38 \%$ at the end of 2000 .

At the end of $2004,28 \%$ of candidates on the waiting list were inactive, including $34 \%$ of those aged 50 years or older. Remarkably, $47 \%$ of the inactive waiting list patients had been waiting for more than 2 years and $73 \%$ for more than 1 year. The high frequency of inactive candidates raises questions regarding the ultimate suitability of these patients for transplantation. It also may reflect the increasing age distribution of the waiting list, and increased complication rates in diabetics as they wait for a pancreas transplant, causing increased frequency of need for temporary inactivation. The annual death rate on the SPK waiting list increased from 88 per 1000 patient-years at risk in 2003 to 95 per 1000 in 2004, the highest rate in the past decade. The increase in death rates also may be attributable to the increasing age of candidates.

The median time to SPK transplant decreased from a peak of 542 days for registrants listed in 2000 to 457 days for 
registrants listed in 2003. Like waiting times for kidney transplantation, time to SPK transplant remains longer for registrants with blood types $O$ and $B$ than for those with blood types $A$ and $A B$, and longer for women than men. The median time to transplant for registrants listed in 2003 increased somewhat with increased age, with registrants ages 18-34 having a considerably lower median time to transplant than registrants ages 50-64 (374 days vs. 530 days). Similarly, the median time to transplant is substantially longer for those listed following a previous organ transplant: 475 days for those without a prior organ transplant and 1174 in 2001 for those with a previous organ transplant (the last year in which both of these figures can be calculated).

\section{Characteristics of SPK transplant recipients}

The number of SPK transplants performed in 2004, at 880 , remains well below the peak of 972 in 1998, but slightly increased from the 870 procedures performed in 2003 (Figure 7). Since 1998, the decline in the number of SPK transplants has been accompanied by an increase in PAK transplants. However, in 2004 the number of transplants in all categories increased over the previous year, indicating an increase in overall pancreas utilization.

The age distribution of recipients parallels that of waiting list registrants. The percentage of SPK recipients who were 50 years of age or older increased from 15\% in 2003 to $19 \%$ in 2004 . Although the majority of recipients of SPK transplants in 2004 were white (75\%), the percentage who were African American increased to 14\% in 2004 (from 9\% in 2000), and the percentage who were Hispanic/Latino increased to $9 \%$ (from $5 \%$ in 2000). SPK recipients continued to receive substantially mismatched organs, partly because most pancreata are not allocated based on HLA matching. Only $10 \%$ of recipients had zero A mismatches, $3 \%$ had zero B mismatches and $8 \%$ had zero DR mismatches. Overall, $94 \%$ of recipients had three or more HLA mismatches and only $1.4 \%$ received a zero mismatch trans-

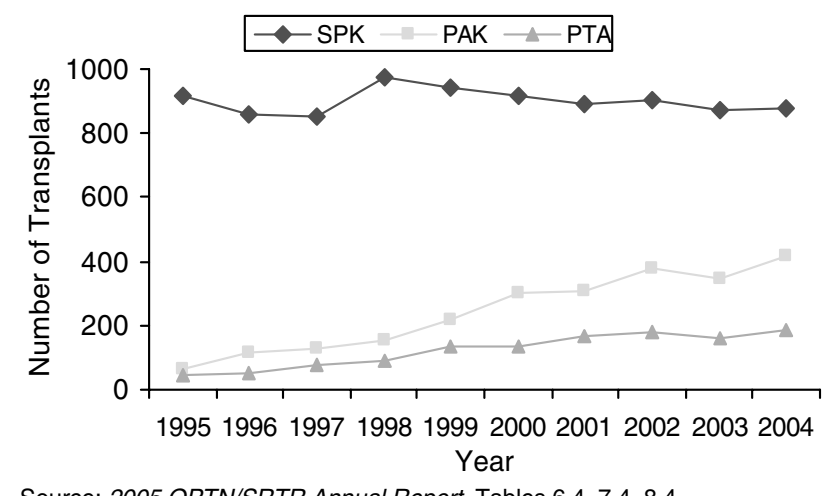

Source: 2005 OPTN/SRTR Annual Report, Tables 6.4, 7.4, 8.4.

Figure 7: Pancreas transplants, by transplant type, 19942005.

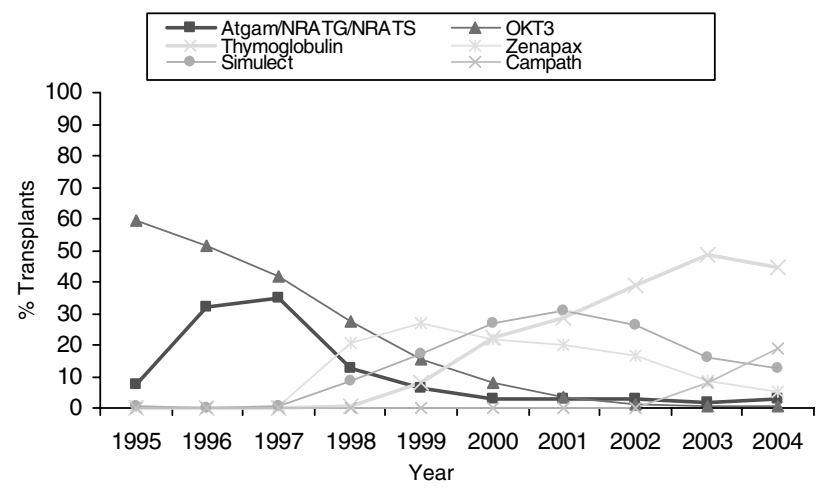

Source: 2005 OPTN/SRTR Annual Report, Table 8.6a.

Figure 8: Immunosuppression agents used in kidneypancreas transplantation, 1995-2004.

plant. CITs for SPK transplants remained stable, with an equal number of transplants performed with CITs of less than $11 \mathrm{~h}$ and CITs of $11-22 \mathrm{~h}$. Less than $5 \%$ of SPK transplants had CITs greater than $22 \mathrm{~h}$. Only $1 \%$ of SPK recipients (compared to $5 \%$ of the waiting list) were previous KP recipients, and $7 \%$ of recipients (compared to $14 \%$ of the waiting list) received SPK transplants following any other organ transplant.

Immunosuppression for SPK transplantation has evolved over the past decade (Figure 8). The frequency of induction increased from $64 \%$ in 1998 to $80 \%$ in 2004 . Prior to 1998, a majority of recipients received either muromonabCD3 or ATG. In 2004, however, $44 \%$ of recipients received rabbit ATG, 19\% received alemtuzumab, 18\% received either basiliximab (Simulect ${ }^{\circledR}$, Novartis, East Hanover, NJ) or daclizumab (Zenapax ${ }^{\circledR}$, Roche, Nutley, NJ) and $21 \%$ did not receive induction.

The majority of recipients $(65 \%)$ received tacrolimus, mycophenolate and steroids as initial maintenance immunosuppression. Only $6 \%$ of recipients were on cyclosporinebased immunosuppression, and $17 \%$ were receiving sirolimus. The percentage of recipients on steroid-free maintenance immunosuppression has increased from $4 \%$ in 2000 to $24 \%$ in 2004 . This increase appears to correspond to the increase in use of depleting induction agents such as rabbit ATG and alemtuzumab.

Eighteen percent of recipients who received transplants in 2003 were treated for rejection in the year following their transplant, compared with $30 \%$ in 1998. The decrease in rejection may be related to increased use of induction, which has been associated with lower rejection rates, although not with increased graft survival, in several multicenter randomized trials $(9,10)$. The decline in rejection may also be a consequence of more accurate methods of diagnosis, such as the increasing use of biopsy (11). Use of steroids in the treatment of rejection is decreasing, down 
to $69 \%$ for those receiving transplants in 2003. Rabbit ATG was used in $40 \%$ of recipients, muromonab-CD3 (OKT3) in $10 \%$, basiliximab and daclizumab in $13 \%$ and alemtuzumab in $4 \%$.

\section{SPK graft survival: kidney}

Unadjusted kidney graft survival rates at 1 year, 3 years and 5 years after SPK transplantation were 92\%, 85\% and $77 \%$, respectively. The rates are somewhat better than unadjusted non-ECD kidney-alone survival rates in diabetics, which were $89 \%, 77 \%$ and $65 \%$, perhaps due to more stringent selection criteria applied to SPK recipients and donors, and shorter CIT. African Americans had worse graft survival rates than whites at all time points; at 5 years graft survival among African Americans was $64 \%$, compared to $78 \%$ among whites. SPK recipients with prior kidney, pancreas or SPK transplants had worse kidney graft survival rates at all time points. The 5-year graft survival rate from donors aged 50-64 years was $67 \%$, compared with a rate of approximately $79 \%$ from donors aged 11-34 years. CIT had virtually no influence on 5 -year graft survival: $75 \%$ for kidneys with CITs of $22-31 \mathrm{~h}$ compared to $78 \%$ for those with CITs of $21 \mathrm{~h}$ or less. Neither center volume nor degree of HLA match was associated with unadjusted 5-year kidney graft survival. Unadjusted 1-, 3- and 5-year kidney graft survival rates have improved only slightly compared to 1996 $(92 \%, 85 \%$ and $75 \%$ in 2004 vs. $89 \%, 82 \%$ and $73 \%$ in 1995).

\section{SPK graft survival: pancreas}

Because pancreas graft failure is defined by report of graft loss by transplant centers to the OPTN, graft survival may not be equivalent to insulin independence. Unadjusted pancreas graft survival rates at 1, 3 and 5 years following SPK transplantation were comparable to the previous year-at $86 \%, 79 \%$ and $71 \%$, respectively (Figure 9). One-year and 3-year graft survival rates increased by $2-3 \%$ over the previous 5 years, while 5 -year graft survival remained unchanged. African American recipients had poorer 5 -year pancreas graft survival (64\%) than all other races/ethnicities (72-75\%). Five-year graft survival was modestly lower among recipients aged 1834 years $(68 \%)$ than among those aged $35-49$ years $(73 \%)$ or 50-64 years $(70 \%)$. On the other hand, 5-year pancreas graft survival after transplants from donors aged 35 years and older (approximately 65\%) was substantially lower than after transplants from donors younger than 35 years (approximately 75\%). As noted above for kidney graft survival rates, recipients with any previous transplant (kidney, pancreas or both) and sensitized recipients had worse pancreas graft outcomes. There continues to be a trend toward decreased 5-year pancreas graft survival with increasing levels of HLA mismatch. As with kidney graft survival in these recipients, pancreas graft survival did not exhibit large differences by transplant center volume.

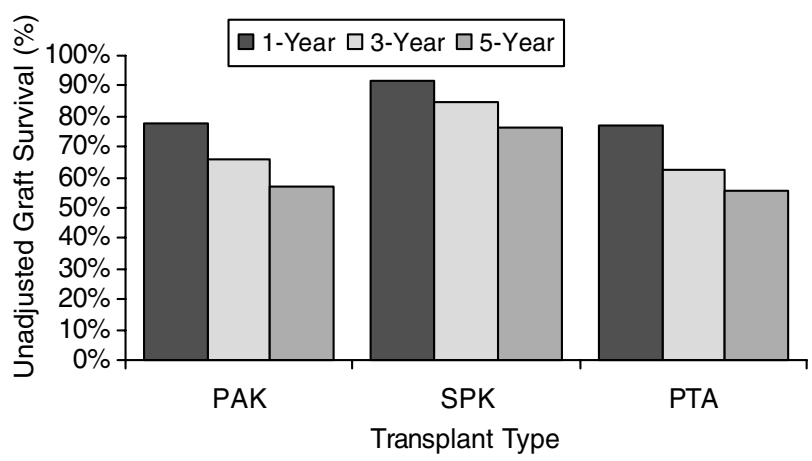

Source: 2005 OPTN/SRTR Annual Report, Tables 6.9, 7.9, 8.9.

Figure 9: Unadjusted 1-year, 3-year and 5-year pancreas graft survival, by transplant type, for transplants received 19982003.

\section{SPK transplant: patient survival}

Patient survival following SPK transplantation is excellent and has increased incrementally since 1995 . There were 7213 recipients of SPK transplants alive with functioning grafts at the end of 2004. Death rates for recipients in the first year following transplant have decreased, from 60 per 1000 patient-years at risk in 2001 to 45 per 1000 in 2003. These rates continue to be lower than the corresponding death rates for candidates on the SPK waiting list. Death rates in the first year following transplantation were similar among ethnicities. Recipients who were 50-64 years old had higher death rates following transplantation than did younger recipients, although these rates were still lower than rates among candidates on the waiting list.

Unadjusted patient survival rates at 1 year (cohort of transplants performed in 2002-2003), 3 years (cohort of transplants performed in 2000-2003) and 5 years (cohort of transplants performed in 1998-2003) were 95\%, 91\% and $86 \%$, respectively. African Americans had only minimally lower (by 4-6\%) unadjusted 5-year survival compared with other ethnic groups, as did recipients age 50 years or older (by 5-7\%) compared with younger recipients. As with kidney graft survival, patient survival in recipients of SPK transplants decreased with increasing donor age over 35 years, which illustrates the relationship between renal function and survival that has been consistently demonstrated in candidates for and recipients of pancreas transplants $(12,13)$. Center volume and HLA mismatch did not exert any influence over patient survival rates.

\section{Pancreas Transplantation Alone and Pancreas After Kidney Transplantation}

\section{The pancreas waiting list}

The number of patients active on the waiting list at the end of the year for isolated pancreas transplants decreased in 


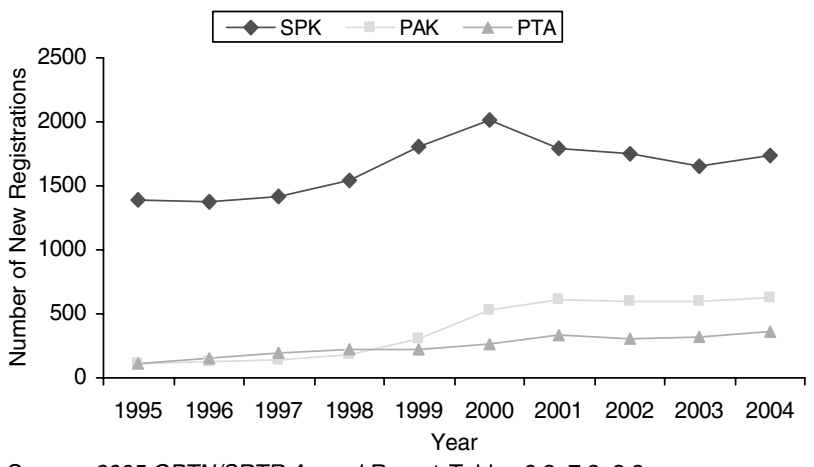

Source: 2005 OPTN/SRTR Annual Report, Tables 6.2, 7.2, 8.2.

Figure 10: New registrations on pancreas waiting list, by transplant type, 1995-2004.

2004, with 420 candidates awaiting a PAK transplant and 265 candidates awaiting pancreas transplantation alone (PTA) (for diabetics without renal failure). At the end of 2003, 534 patients were awaiting PAK and 303 patients were awaiting PTA. Although the number of patients listed for isolated pancreas transplant decreased between 2003 and 2004, the number of new registrations increased for both PAK (from 591 to 620) and PTA (from 324 to 364) (Figure 10). While the great majority of patients awaiting isolated pancreas transplants are white (81\% for PAK, 89\% for PTA), the percentage of white patients has decreased over the last several years, particularly for PAK. The increasing representation of non-whites may be related to increased access as a result of expanded insurance coverage. Although the majority of patients awaiting both PAK and PTA were between 35 and 49 years old in 2004, 17\% of active PAK candidates and $16 \%$ of active PTA candidates were age 50 or older, compared with only 10\% and 9\% in 1998. The increase in the number of older candidates has stabilized over the past few years. Death rates among patients on the PAK and PTA waiting lists are highly variable from year to year due to the small number of events, but they tend to be higher on the PTA waiting list. Among candidates on the waiting list for PTA in 2004, the death rate was 53 per 1000 patient-years at risk, while the death rate among waiting list candidates for PAK was 27 per 1000 patientyears at risk. A large proportion of candidates waitlisted for PAK (57\%) and PTA (47\%) at the end of 2004 were not active; this was a substantial increase from 2000-2003. The reasons for the increasingly high proportion of inactive candidates are not known. As seen with candidates for SPK, a disproportionate percentage of inactive waitlisted PTA and PAK candidates had been waiting more than 2 years at the end of $2004(67 \%$ and $47 \%$ inactive vs. $20 \%$ and $26 \%$ active patients).

The number of solitary pancreas transplants increased dramatically in 2004, with 419 PAK and 185 PTA transplants performed. This is an $18 \%$ increase in pancreas utilization for PAK and a 14\% increase in PTA since 2003. The median time to transplant was 504 days for PAK and 348 days for PTA for 2003 registrants, compared with 590 days for PAK and 302 days for PTA for 2002 registrants. The shorter waiting times for PTAs suggest that solitary pancreata are more readily available than SPKs. In some donation service areas (DSAs) this may reflect policies that prioritize solitary pancreas allocation and kidney-alone allocation over SPK for certain candidates. However, some DSAs have changed their allocation policies to favor SPK candidates, in recognition of the value of renal function to pancreas candidates. Eight percent of PAK and 6\% of PTA transplants were performed with CIT greater than $22 \mathrm{~h}$, which likely reflects the increased number of solitary pancreata that are shared nationally (M.D. Stegall et al. unpublished data, 2005).

Previous increases in the number of patients listed for isolated pancreas transplant likely reflected improved results, the increase in the number of people receiving living donor kidneys, and expanded insurance coverage. Although this trend continues in an increasing number of new registrants, it appears that solitary pancreas utilization is increasing to match the increase in registrants, with a consequent decrease in the number of active waiting list candidates.

\section{Recipient characteristics and outcomes of PAK transplantation}

There continues to be significant uncertainty in the transplant community about whether solitary pancreas transplantation, either PTA or PAK, provides a survival advantage. Similar analyses by Venstrom (12) and Gruessner (13), while demonstrating a conclusive benefit of SPK transplantation versus remaining listed for SPK, drew different conclusions regarding the survival benefit of solitary pancreas transplantation. The differences between these two studies lie primarily in the determination of waiting list mortality, specifically in the censoring of waiting list removals and candidates with renal insufficiency. The fact that different outcomes resulted from these subtle differences in methodology probably signifies that any effect, whether beneficial or adverse, of pancreas transplantation on mortality is likely to be small. Nevertheless, this debate has not apparently had an adverse impact on new candidates or transplant volume.

The age and race of recipients of PAK transplants in 2004 are reflective of the distribution of waiting list registrants. In $2004,87 \%$ of recipients were white and $58 \%$ were male. Age at transplant remained relatively consistent over the previous 5 years, with a slight shift to older patients receiving PAK. In 2004, $21 \%$ of the patients were between the ages of 18 and $34,64 \%$ were between the ages of 35 and 49 and $15 \%$ were between the ages of 50 and 64 . Twenty-three percent of recipients of PAK had received a pancreas transplant previously.

The frequency of induction for PAK is similar to SPK; $80 \%$ received some form of induction therapy. A larger 
percentage of PAK recipients received a lymphocytedepleting agent, either rabbit ATG (58\%) or alemtuzumab (20\%). Recipients received basiliximab or daclizumab in $12 \%$ of cases, less than for SPK. The vast majority of recipients received tacrolimus, mycophenolate and steroids as maintenance immunosuppression at the time of hospital discharge. The frequency of immunosuppressive agents used for maintenance immunosuppression was similar to that for SPK, including the proportion not receiving steroids, as was the 1-year rejection rate of $18 \%$ for those receiving transplants in 2003.

Unadjusted graft survival rates for PAK at 1 year, 3 years and 5 years were $78 \%, 66 \%$ and $57 \%$, respectively (Figure 9). There was $7 \%$ graft loss during the initial hospitalization. The 1-year unadjusted pancreas graft survival rate for PAK transplants did not change between 2002 and 2003. Unadjusted 3-year and 5-year survival rates increased by $16 \%$ and $4 \%$, respectively, compared to 1995 , suggesting that perhaps long-term success rates will improve with longer follow-up.

The unadjusted patient survival rates for PAK recipients at 1 year, 3 years and 5 years were 96\%, 90\% and 84\%, respectively. These were unchanged from the results presented in the 2004 OPTN/SRTR Annual Report. The annual death rate per 1000 patient-years at risk for recipients in the first year following PAK transplantation has been consistent since 1999 and was 51 for 2003.

\section{Recipient characteristics and outcomes of PTA}

The age distribution of PTA recipients parallels that of the waiting list: $22 \%$ aged $18-34$ years, $47 \%$ aged $35-49$ years and $15 \%$ aged $50-64$ years. Over the past decade, there was a trend favoring older recipients. The race and sex of recipients of PTA in 2004 also reflected the distribution of registrants on the waiting list. Fifty-eight percent of recipients were female and $90 \%$ were white. The percentage of non-white PTA recipients ranged from $4 \%$ to $14 \%$ annually during the past decade. Interestingly, $12 \%$ of PTA candidates had received prior pancreas or pancreas-kidney transplants, and $14 \%$ had previously received other organ transplants.

In 2004, 51\% of PTA recipients received rabbit ATG induction, $43 \%$ received alemtuzumab and $9 \%$ received daclizumab. Only $77 \%$ of recipients in 2004 received calcineurin inhibitors, primarily tacrolimus, for maintenance immunosuppression and only 50\% received steroids. These data are undoubtedly influenced by transplant center effects to a greater degree than are data in other pancreas transplant categories, because much of the national PTA experience is concentrated in a small number of centers. Despite a reduced reliance on steroids and/or calcineurin inhibitors, the rejection rate for PTAs performed in 1993 was 16\%, similar to other pancreas transplant categories.
The unadjusted graft survival rates for PTA at 1, 3 and 5 years in 2004 were $77 \%, 62 \%$ and $56 \%$, respectively, representing significant improvement since 1995. Graft survival was somewhat better in older PTA and PAK recipients. There was a trend toward poor graft outcome with increasing donor age at 1 year and 3 years, but not at 5 years. Overall, the results of PTA now approximate those observed for PAK. Patient survival following PTA was the highest of all pancreas transplant categories. The unadjusted patient survival rates for PTA recipients at 1 year, 3 years and 5 years were $96 \%, 94 \%$ and $91 \%$.

\section{Pancreas Transplant Utilization}

Although the number of whole organ pancreas transplants performed increased substantially in 2004, there is broad agreement that the efficiency of pancreas allocation can still be enhanced to promote increased utilization for both whole organ and islet transplantation. This view was the basis for changes in the pancreas allocation algorithm that were adopted by the OPTN Board in June 2004. The new pancreas allocation policies, and contemporaneous changes in islet program certification criteria that were also adopted by the OPTN, were reviewed and generally supported at the OPTN Pancreas Allocation Consensus Conference in January 2005 (M.D. Stegall et al. unpublished data, 2005). In preparation for this conference, members of the OPTN Kidney Pancreas Committee, in conjunction with the SRTR and UNOS, studied the utilization of deceased donor pancreata from 2000 to 2003 (M.D. Stegall et al. unpublished data, 2005). During that time period, 24 703 potential pancreas donors were identified. Consent was obtained, but pancreata were not recovered from $48 \%$ (11 820) of donors, mostly for reasons related to donor quality. Of the 9094 pancreata recovered for the purpose of whole organ transplantation, islet transplantation or research, 5521 resulted in a whole organ pancreas transplant and 1052 were discarded. The majority of donors (58\%) were younger than age 50 with a body mass index (BMI) less than $30 \mathrm{~kg} / \mathrm{m} 2$; these donors accounted for $90 \%$ of the transplants performed. Nevertheless, pancreata were not recovered from $52 \%$ of these donors, and the rate of pancreatic recovery varied widely (from $24 \%$ to $50 \%$ ) among UNOS regions. These data suggest potential for increased utilization of pancreata for whole organ transplant, and that the recent OPTN policy that allocates pancreata from donors older than age 50 with BMI greater than $30 \mathrm{~kg} / \mathrm{m} 2$ for islet transplantation is unlikely to have an adverse impact on pancreas utilization for whole organ transplantation.

\section{Kidney-Pancreas Allocation: Policy Update}

In 2004, the OPTN Board of Directors approved several policy changes affecting KP allocation (14). These changes relate to pediatric transplant candidates, living donor/list 
exchanges, an alternate system to calculate waiting time for waitlisted kidney transplant candidates, sharing algorithms, and islet cell transplantation. The impact of these changes is not reflected in the data presented in this article because several of the policy changes have yet to be implemented.

The intent of the changes in the allocation policies regarding pediatric patients is to facilitate transplantation for pediatric candidates. Children have long been recognized as deserving priority in the allocation of kidneys because of the adverse effects of ESRD on growth and development. Children will receive priority for SCD kidneys from donors less than 35 years of age, behind only zero-antigen mismatches, those with PRA of $80 \%$ or higher, combined kidney/nonrenal organ candidates and prior organ donors. This policy also benefits the high-PRA patients who, along with pediatric patients, will now have priority for local kidneys over paybacks (OPTN Policies 3.5.5 and 3.5.11.5.1).

Changes in distribution algorithms include increasing the ABO blood group payback debt threshold from four to six, thereby facilitating an organ procurement organization's (OPO's) ability to obtain and retain kidneys to be used in SPK transplantation. Similarly, the requirements for sharing zero-mismatched isolated pancreata are eliminated under all circumstances except in the case of a potential recipient with a PRA greater than $80 \%$. These allocation changes are designed to minimize obstacles to the use of kidneys and pancreata from the same donor for SPK transplantation (OPTN Policies 3.5.5.1.1, 3.5.5.1.2 and 3.8.1.6).

Other modifications include an exemption from mandatory zero-antigen mismatch sharing (except at the local level) and payback requirements for DCD kidneys. These exemptions are intended to minimize CIT for DCD kidneys and to encourage centers to develop DCD donor programs. Similarly, ECD kidneys are exempted from kidney payback policy (OPTN Policies 3.5.3.3 and 3.5.5).

The OPTN Board of Directors also approved a priority system in the event that there are multiple waitlisted candidates with incompatible living kidney donors who donated to the list so that their intended recipients would receive priority. In this case, the intended recipient whose incompatible donor donated to the list earlier would receive priority for an appropriate available kidney.

Modification to the OPTN Policy 3.8.1.5 is intended to prevent waste of donated pancreata by permitting an islet preparation that is deemed unsuitable for the candidate on top of the list to be used for a different, more suitable candidate on the waiting list.

Finally, a voluntary study was initiated to assess the effects of an alternative system for calculating waiting time for waitlisted candidates. In place of a first-come, first-served approach for listing patients who meet minimum listing criteria, this new system would calculate waiting time from the earlier of the time that the patient began maintenance hemodialysis or the time that the calculated or measured glomerular filtration rate (GFR) was less than $20 \mathrm{ml} / \mathrm{min}$, rather than the time the patient happened to be referred to the transplant center and completed his/her evaluation.

\section{Emerging Issues in Donation After Cardiac Death}

The shortage of organ donors has led the transplant community to pursue multiple avenues to increase the pool of potential donors. Although the full potential for maximizing the donor pool has not been met, efforts to date have been successful, with dramatic increases in the number of living kidney donors over the past decade and, more recently, an increase in deceased kidney donors. Increases in living kidney donors have been achieved through the development of laparoscopic nephrectomy, the acceptance of emotionally related living donors, paired live donor donation, non-directed donation and intended candidate exchanges with the waiting list. Increased deceased donor kidney availability has been accomplished through public education campaigns, the use of ECD kidneys, the realization that kidneys from Hepatitis C-infected donors can be transplanted safely into Hepatitis $\mathrm{C}$-infected recipients, the use of two kidneys from a deceased donor with suboptimal renal function into a single recipient, and now, most recently, the use of donation after cardiac death. As noted above, the most rapidly growing source of kidney transplants is DCD donors; these transplants increased 39\% between 2003 and 2004 .

Published reports on the successful use of solid organ transplantation from 'non-heart-beating donors' began to appear in the early to mid-1990s. These reports included data from programs that began as early as 1980 . With follow-up periods ranging from 1 to 9 years, results obtained were often equal to those obtained with standard brain dead, heart-beating donors. When inferior, the results were still quite respectable. In 1998, Cho et al. reported on 229 patients from the UNOS Registry (15). Again, results were comparable to those obtained from heart-beating donors, despite the higher incidence of DGF and a higher primary failure rate. The authors speculated that the number of patients on the waiting list would remain constant if each OPO obtained two kidneys each month from previously unused non-heart-beating donors. These reports were summarized by Weber et al. with follow-up from another 122 patients in 2002 (16). By 2002, it had become clear, as discussed by Cecka in an editorial accompanying the Weber paper, that non-heart-beating donation had the potential to substantially increase the pool of kidneys suitable for transplantation $(16,17)$. At the same time that the Institute of Medicine, the American Society of Critical Care 


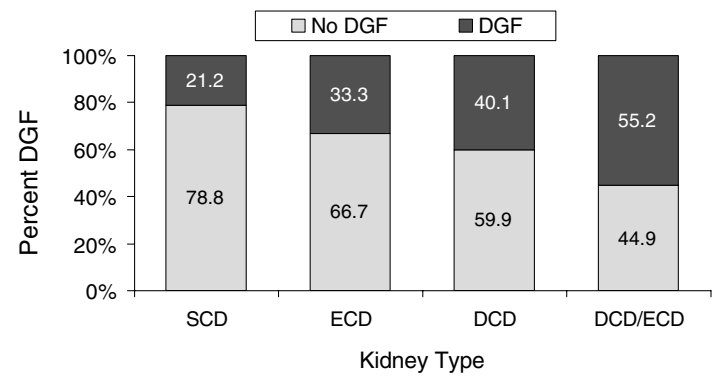

There were 454/41,218 non-DCD and 27/1,635 DCD kidneys with missing DGF information.

Source: SRTR Analysis, April 2005.

Figure 11: Delayed graft function (DGF) for DCD vs. Non-DCD kidneys (with and without ECD), 2000-2004.

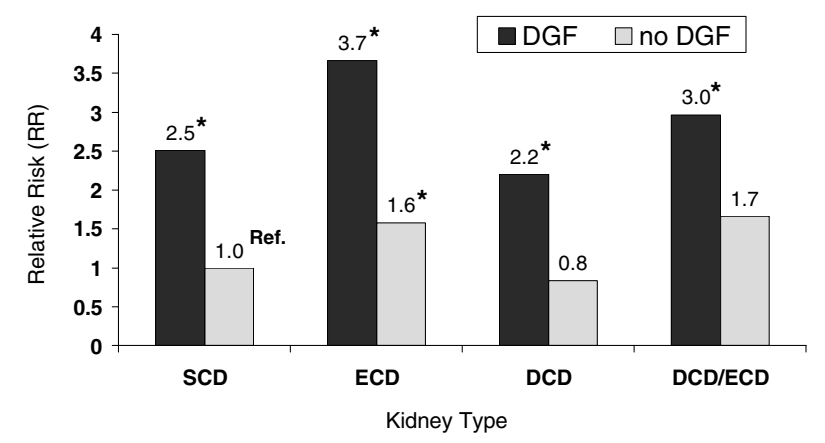

${ }^{*} \mathrm{P}<0.05$; interactions were not statistically significant. Source: SRTR Analysis, April 2005.

Figure 12: Adjusted relative risk (RR) of graft failure by delayed graft function (DGF) and donor type (interaction), 20002004.

Medicine, and the Joint Commission on Accreditation of Healthcare Organizations all reached the conclusion that organ procurement after cessation of heartbeat was ethically acceptable provided that proper protocols were in place, an increasing number of OPOs reported procuring such organs (18-20).
There were only 16 OPOs reporting the transplantation of organs procured from DCD donors in 1998. This number rose to 31 in 2002 and 41 in 2004 . The use of such donors as a percentage of total OPO activity ranged from $0 \%$ to $20 \%$ in 2004 . Nearly one third of all DSAs continue to report having no DCD organs. In contrast, in The Netherlands, one third of all kidneys transplanted derive from DCD donors (21). The Advisory Committee on Organ Transplantation to the U.S. Secretary of Health and Human Services recommended that each hospital, as a condition of participation with the Centers for Medicare and Medicaid Services, 'establish, in conjunction with its OPO, policies and procedures to manage and maximize organ retrieval from donors without a heartbeat' (22).

As with donation after brain death, it is important to consider results of transplantation using organs procured from DCD donors based on the quality of the organ transplanted. As noted in Figure 11, DGF remains more frequent with DCD kidneys compared to SCD kidneys; DCD kidneys that meet ECD criteria (DCD/ECD) experience DGF in 55\% of cases. The presence of DGF is associated with an elevated risk of graft failure for each category of donor (Figure 12). However, the added relative risk of graft failure resulting from DGF is similar across all the groups. Table 2 shows that the extension of DCD protocols to additional OPOs has not compromised results. The factor demonstrating the greatest influence on graft survival is not whether the kidney derives from a DCD or SCD donor, but whether the kidney meets ECD criteria. Outcomes of kidney transplants from DCD donors who do not meet ECD criteria are comparable to SCD kidney transplant outcomes, despite double the percentage of DGF, while outcomes of transplantation from DCD donors who do meet the ECD definition (DCD/ECD in Table 2) are similar to those of ECD/non-DCD kidney transplants.

Despite the unquestionable success in use of DCD donor kidneys, this is a rapidly evolving area with many unresolved medical, ethical and legal issues. The selection of patients for whom withdrawal of therapy is planned and DCD is being considered, and the protocols for their care, need refinement. Protocols for determining the

Table 2: Adjusted ${ }^{1}$ 1-year and 3-year kidney graft survival rates, by donor type and percentage with delayed graft function (DGF), 2000-2004

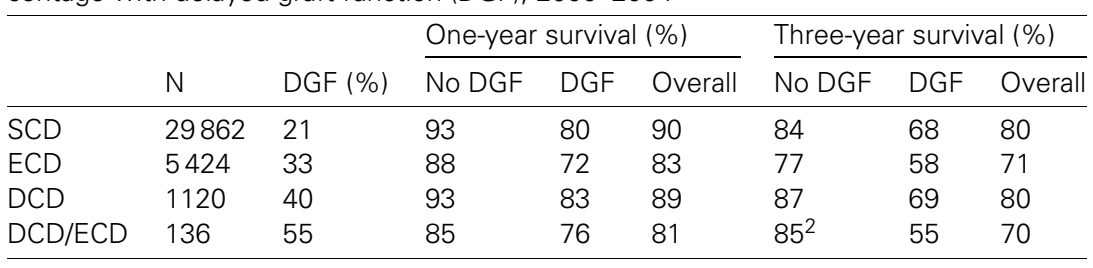

Source: SRTR Analysis, April 2005.

${ }^{1}$ Adjusted for recipient age, sex, race, PRA, ESRD cause, years of ESRD, HLA mismatch, year of transplant, previous transplant, transfusions and donor sex, race, diabetes, cold ischemia time.

${ }^{2}$ No patients in this group after Day 313. 
irreversibility of the cessation of circulatory and respiratory function, including the period of observation from asystole to the determination of death, remain unstandardized. In addition, the use of medications intended to improve posttransplant organ function, but not specifically related to end-of-life care of the donor, remains controversial, as unintentional hastening of death may occur. It is also unconfirmed whether pre-procurement treatments truly improve posttransplant outcomes. Finally, the public perception of the increased used of DCD donors and the effect that such practices might have on donation after brain death must be a constant focus so that the net effect is an increase, not a decrease, in transplantable organs. This is particularly true for 'uncontrolled' DCD, little used at present, which may require pre-procurement and even pre-consent treatments, in order for successful organ donation to occur. As stated in the report of the 2005 National Conference on Donation after Cardiac Death, the top priority in care for a potential organ donor must always be quality end-of-life care, and the donation process should never compromise this quality (Bernat et al. unpublished report, 2005). The opportunity for DCD organ donation should, under appropriate circumstances, be viewed as a routine option for families considering withdrawal of life support.

\section{Acknowledgment}

The Scientific Registry of Transplant Recipients is funded by contract number 231-00-0116 from the Health Resources and Services Administration (HRSA), U.S. Department of Health and Human Services. The views expressed herein are those of the authors and not necessarily those of the U.S. Government. This is a U.S. Government-sponsored work. There are no restrictions on its use.

This study was approved by HRSA's SRTR project officer. HRSA has determined that this study satisfies the criteria for the IRB exemption described in the "Public Benefit and Service Program" provisions of 45 CFR 46.101 (b)(5) and HRSA Circular 03.

\section{References}

1. Levine GN, McCullough KP, Rodgers AM, Dickinson DM, Ashby VB, Schaubel DE. Analytical methods and database design: Implications for transplant researchers, 2005. Am J Transplant 2006; 6(Part 2): 1228-1242.

2. U.S. Renal Data System. 2004 Annual Data Report: Atlas of EndStage Renal Disease in the United States. Bethesda, MD: Nationa Institutes of Health, National Institute of Diabetes and Digestive and Kidney Diseases, 2004

3. U.S. Bureau of the Census. U.S. Summary: 2000 Census Profile. Washington, DC: U.S. Census Bureau, 2002.

4. Nelson PW, Shield CF, 3rd, Muruve NA, Murillo D, Warady BA, Aeder MI. Increased access to transplantation for blood group B cadaveric waiting list candidates by using A2 kidneys: Time for a new national system? Am J Transplant 2002; 2: 94-99.
5. Newell KAWM, Guidinger MK, Sung RS. Pre-emptive renal transplantation: Does everyone benefit? (abstract). Am J Transplant 2005; 5(Suppl 11): 293.

6. U.S. Bureau of the Census. Current Population Reports, Series P23-189, Population Profiles of the United States: 1995. Washington, DC: U.S. Government Printing Office, 1995.

7. Bunnapradist S, Cho YW, Cecka JM, Wilkinson A, Danovitch GM Kidney allograft and patient survival in type I diabetic recipients of cadaveric kidney alone versus simultaneous pancreas kidney transplants: A multivariate analysis of the UNOS database. J Am Soc Nephrol 2003; 14: 208-213.

8. Israni AK, Feldman HI, Propert KJ, Leonard M, Mange KC. Impact of simultaneous kidney-pancreas transplant and timing of transplant on kidney allograft survival. Am J Transplant 2005; 5: 374-382.

9. Kaufman DB, lii GW, Bruce DS, Johnson CP, Gaber AO, Sutherland $\mathrm{DE}$ et al. Prospective, randomized, multi-center trial of antibody induction therapy in simultaneous pancreas-kidney transplantation. Am J Transplant 2003; 3: 855-864.

10. Stratta RJ, Alloway RR, Lo A, Hodge E. Two-dose daclizumab regimen in simultaneous kidney-pancreas transplant recipients: Primary endpoint analysis of a multicenter, randomized study. Transplantation 2003; 75: 1260-1266.

11. Kuo PC, Johnson LB, Schweitzer EJ, Klassen DK, Hoehn-Saric EW, Weir MR et al. Solitary pancreas allografts. The role of percutaneous biopsy and standardized histologic grading of rejection. Arch Surg 1997; 132: 52-57.

12. Venstrom JM, McBride MA, Rother KI, Hirshberg B, Orchard TJ, Harlan DM. Survival after pancreas transplantation in patients with diabetes and preserved kidney function. JAMA 2003; 290: 28172823.

13. Gruessner RW, Sutherland DE, Gruessner AC. Mortality assessment for pancreas transplants. Am J Transplant 2004; 4: 20182026.

14. http://www.unos.org/PoliciesandBylaws/policies/docs/policy_9. doc. Accessed 24 August 2005

15. Cho YW, Terasaki PI, Cecka JM, Gjertson DW. Transplantation of kidneys from donors whose hearts have stopped beating. N Engl J Med 1998; 338: 221-225.

16. Weber M, Dindo D, Demartines N, Ambuhl PM, Clavien PA. Kidney transplantation from donors without a heartbeat. N Engl J Med 2002; 347: 248-255

17. Cecka JM. Donors without a heartbeat. N Engl J Med 2002; 347: 281-283.

18. Institute of Medicine. Non-Heart-Beating Organ Transplantation: Medical and Ethical Issues in Procurement. Washington, DC: National Academy Press, 1997.

19. Institute of Medicine. Committee on Non-Heart-Beating Transplantation II. Non-Heart-Beating Organ Transplantation: Practice and Protocols. Washington, DC: National Academy Press, 2000.

20. Joint Commission on Accreditation of Healthcare Organizations. Health Care at the Crossroads: Strategies for Narrowing the Organ Donation Gap and Protecting Patients. Oakbrook Terrace, IL, 2004.

21. Bos MA. Ethical and legal issues in non-heart-beating organ donation. Transplantation 2005; 79: 1143-1147.

22. http://www.organdonor.gov/acotrecsbrief.html. Accessed 24 August 2005 